Indonesian Journal of Cardiology

Indonesian J Cardiol 202 1:42:70-75

pISSN: 0I 26-3773 / elSSN: 2620-4762

doi: $10.30701 /$ ijc. 1152

\title{
Patent Foramen Ovale Implying Paradoxical Embolism as a New Insight in Cryptogenic Stroke
}

\author{
Indah Aprianti Putri
}

National Brain Centre Hospital, Jakarta, Indonesia

\section{Correspondence:}

Indah Aprianti Putri,

National Brain Centre Hospital, Jakarta, Indonesia

Email: iapriantiputri@gmail.com

\begin{abstract}
Background: Cerebrovascular thromboembolism is responsible annually for 510.000 ischaemic stroke in the United States alone. Patent foramen ovale mechanism as a paradoxical embolism transit from right to left-sided chambers to intracranial vessels has a tremendous impact in neurological deficits. The aggressive treatment started since 2016 when the US Food and Drug Administration (FDA) approved the Amplatzer patent foramen ovale occluder for recurrent stroke prevention of cryptogenic stroke with patent foramen ovale. The trials show positive results since 2017 and the collaboration and partnership between neurologist and cardiologist are more needed to build a holistic and comprehensive treatment for cryptogenic stroke patient with patent foramen ovale.
\end{abstract}

(Indonesian J Cardiol. 2021;42:70-75)

Keywords: cryptogenic stroke, patent foramen ovale, paradoxical embolism, patent foramen ovale occluder, comprehensive treatment. 


\section{Introduction}

I schaemic stroke is one of the leading causes of mortality and morbidity in the world. Globally, there are more than 1.2 million stroke survivors. It is a devastating disease with clinical manifestations as focal or global neurological deficits. Patients with ischaemic stroke have negative effects on their quality of life with loss of their cognitive capacity and physical capabilities. Stroke survivors need the prevention from recurrence of ischaemic stroke events. ${ }^{1-3}$

Mostly, ischaemic stroke aetiologies are classified according to the Trial of Org 10172 in Acute Stroke Treatment (TOAST) classification. By this classification, usual mechanisms of ischaemic stroke include stroke secondary to large vessel disease (clot at carotid or part of large vessel of intracranial vessels), cardioembolic events, small vessel occlusion (lacunar infarct, leukoariosis and microbleeds), other identified cause (criptogenic stroke or embolic stroke of undetermined source or ESUS) or have no determined cause. ${ }^{4}$

AAnother group of sub-types of ischaemic stroke is ASCOD. This classification system offers objective criteria for Atherosclerosis/atherothrombosis, Small vessel disease, Cardioembolic stroke, Other causes such as criptogenic stroke, and Dissection. ${ }^{4}$

About $20-30 \%$ of cryptogenic strokes are associated with patent foramen ovale. Since 2017, studies have shown significant treatment of patent foramen ovale closure in criptogenic stroke which has implying paradoxical embolism as the mechanism of this stroke in this group of sub-types. This means that a management of team work between neurologist and cardiologist will improve the beneficial treatment for preventing recurrent of ischaemic stroke. ${ }^{4}$

\section{Patent Foramen Ovale in Cryptogenic Stroke (Embolic Stroke of Undetermined Source or ESUS) $)^{5-6}$}

Most criptogenic strokes are embolic, the sources are coming from:

- Cardiogenic embolism such as unrecognized paroxysmal atrial fibrillation or atrial high-rate episodes/atrial systole.

- Paradoxical embolism via patent foramen ovale (23\% of the population) or other right (R) to left (L) intra-cardiac shunt.
- Arteriogenic embolism for example aortic arch atheroma with ulceration or thrombi, non-stenotic ulcerated cervical carotid atheroma, non stenotic intracranial large artery atheroma.

Paradoxical embolism in Criptogenic stroke via patent foramen ovale based on ESUS criteria:

1. Acute brain infarct visualized on neuroimaging that is non lacunar.

2. Absence of occlusive proximal atherosclerosis.

3. No major-risk cardioembolic source with normal heart rhytm.

4. No other likely cause of stroke (such as dissection, arteritis).

\section{Neurological Manifestation to Diagnose Patent Foramen Ovale in Cryptogenic Stroke ${ }^{7}$}

The manifestation of neurological symptoms in criptogenic stroke are based on BAMFORD study ${ }^{6-7}$. The Bamford classification consists of the following types of ischaemic stroke:

- Total Anterior Circulation Infarction (TACI) cortical stroke in middle or anterior cerebral artery territory. Patients are diagnosed by all three of the following:

1. Unilateral weakness (and/or sensory deficit) of face, arm, or leg.

2. Homonymous hemianopia.

3. Higher cerebral dysfunction (dysphasia, visuospatial disorder).

- Partial Anterior Circulation Infarction (PACI) cortical stroke in middle or anterior cerebral artery areas. Patients are diagnosed by two of the following: 1. Unilateral weakness (and/or sensory deficit) of face, arm, or leg.

2. Homonymous hemianopia.

3. Higher cerebral dysfunction (dysphasia, visuospatial disorder).

- Posterior circulation infarction (POCI), the diagnosis is made if the patient has one of the following:

1. Cerebellar or brainstem syndrome.

2. Loss of consciousness.

3. Isolated homonymous hemianopia. 
- Lacunar infarction (LACI) - subcortical stroke due to small vessel disease, there is no evidence of higher cerebral dysfunction. Diagnosed by one of the following symptoms:

1. Pure Motor Stroke.

2. Sensorimotor Stroke.

3. Pure sensory stroke.

4. Ataxic hemiparesis.

5. Dysarthria-clumsy hand.

\section{How to Diagnose Patent Foramen Ovale in Cryptogenic Stroke}

How to diagnose patent foramen ovale in criptogenic stroke need some evidence that in the further diagnostic examination shows that no evidence by the cause related to large vessel, small vessel, cardioembolic stroke such as atrial fibrillation. It is also not related with other causes such as dissection, or others paradoxycal embolism and hypoperfusion.

Patent foramen ovale is a main cause of left circulation of heart thromboembolism in criptogenic stroke with other clues for example young age, within a limited vascular risk factors of the patient history of medical illness. High risk of patent foramen ovale will more suspicious if some evidences such as :

- $\quad$ D dimer $>1000$

- First event of ischaemic stroke with non lacunar infarction from brain CT or MRI

- Absence of cervical carotid atherosclerotic artery stenosis $>50 \%$ or occlusion

- No atrial fibrillation after $>24$ hours cardiac rhytm monitoring

- No intra-cardiac thrombus on echocardiography

- No history of antiplatelet or anticoagulant

- History of hypercoagulable state

- Evidence of patent foramen ovale from TEE bubble

- Large inter-atrial shunt or an strial septum aneurysm (ASA) positive from echo

- TCD bubble test positive with RoPe score need to do to evaluate patient risk of recurrent ischaemic stroke. $^{8-10}$

\section{TCD Bubble Test}

Transcranial Doppler (TCD) is the only real-time technique (Figure 1 and 2) for finding emboli from extra-cranial carotid plaque or cardiac embolism by continuous monitoring and detection of micro-embolic signals or high intensity transient signals (MES or HITS) in cerebral circulation. ${ }^{11}$

MES can be identified as a short lasting $(<0.01$ $0.03 \mathrm{~s}$ ), unidirectional signal with an intensity increase ( $>3 \mathrm{DB}$ ) within the Doppler frequency spectrum. MES appears randomly within the cardiac cycle and produces a" whistling", "chirping" or "clicking" sound when passing through the sample volume. ${ }^{12}$

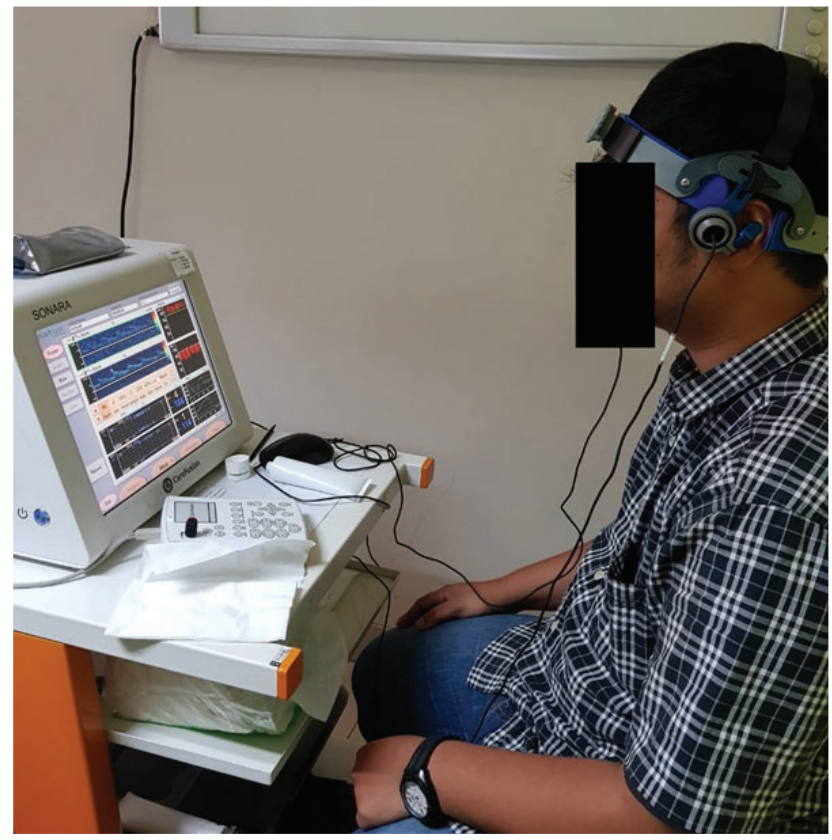

Figure 1. Technique position of a patient undergoing TCD (Courtesy of the Author).

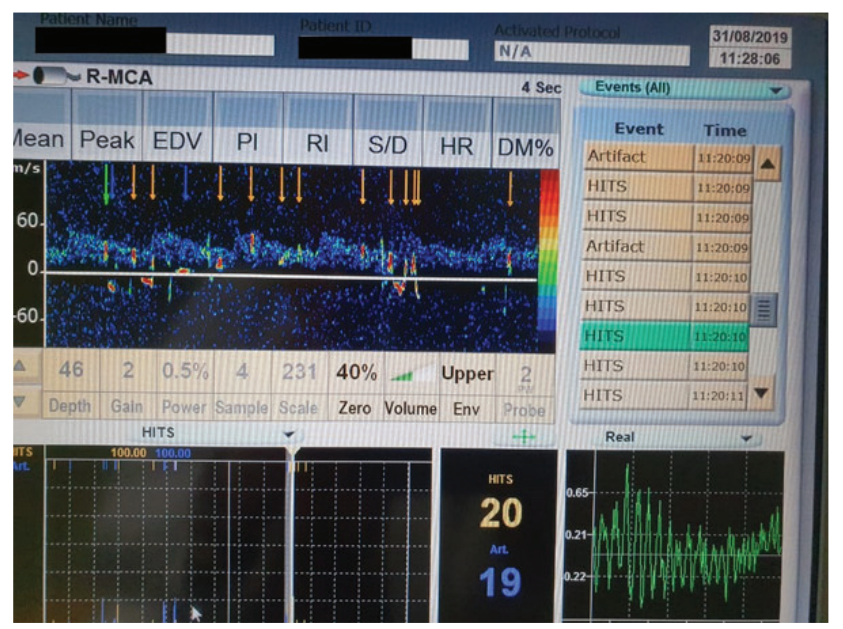

Figure 2. MES or HITS appearances (Courtesy of the author). 
Indonesian Journal of Cardiology

The grading of Spencer is a guidance for neurologist to make a diagnostic of suspected Patent Foramen Ovale in cryptogenic ${ }^{13}$ :

- Grade 0, no microemboli

- Grade 1, 1-10 microemboli

- Grade 2, 11-30 microemboli

- Grade 3, 31-100 microemboli

- Grade 4, 101-300 microemboli

- Grade 5, >300 microemboli

\section{Treatment Patent Foramen Ovale in Cryptogen- ic Stroke}

The treatment of cryptogenic stroke with anticoagulant and antiplatelet based on COMPASS study. Red clot and white clot mechanism of cryptogenic stroke need anticoagulant and antiplatelet to lysis the clot of cryptogenic stroke. ${ }^{14}$

Treatment with rivaroxaban $2.5 \mathrm{mg}$ and aspirin 100 $\mathrm{mg}$ are indicated for reccurent ischaemic stroke in ESUS patients with moderate-severe left atrial enlargement (LA diameter $>4.6 \mathrm{~cm}$ ). Another study is ARCADIA trial, the hypothesis state that apixaban is superior to aspirin for prevention of recurrent stroke in patients with ESUS and atrial cardiopathy. ${ }^{15}$

Percutaneous patent foramen ovale closure has been studied for more than two decades, marked by a series of negative, underpowered and inconclusive trials. In 2016, the US Food and Drug Administration approved the Amplatzer patent foramen ovale occluder for recurrent stroke prevention. Historically, support for patent foramen ovale closure has been stronger among cardiologist-especially interventional cardiologistthan among neurologist. What we knew related into treatment of patent foramen ovale, 3 trials namely Closure trial, PC trial (Percutaneus Closure of patent foramen ovale in criptogenic embolism) and RESPECT trial (Randomized Evaluation of Reccurent Stroke Comparing patent foramen ovale Closure to Established Current Standard of Care Treatment) published before 2017 did not show any benefit of patent foramen ovale closure over medical treatment in reducing recurrence stroke in patients with cryptogenic stroke associated with patent foramen ovale. ${ }^{16}$

CLOSE trials as randomized, open label, superiority trial conducted in Europe. The participants were 16-60 years old and enrolled from December 2007 through December 2014 and followed up until December 2016. The participants had recent ( $\leq 6$ months) cryptogenic stroke attributed to patent foramen ovale with an atrial septum aneurysm or large inter-atrial shunt.

Eligible patients were randomly assigned, in a 1:1: ratio, to undergo patent foramen ovale closure followed by long term antiplatelet therapy (patent foramen ovale closure group), or to receive antiplatelet therapy alone (antiplatelet group), or oral anticoagulant (anticoagulant group). The primary efficacy outcome was the occurance of any stroke. The secondary efficacy outcomes were the composite of ischaemic stroke, transient ischemic attack (TIA) or systemic embolism; disabling stroke (defined as $\mathrm{mRS} \geq 3$ ), death from vascular-related causes; success of device implantation, and success of patent foramen ovale closure. The safety outcomes were procedural or haemorrhagic complications. A clinical events committee blindly adjudicated outcome events. Patients were followed up at 2 months, 6 months, and thereafter 6 months by direct visit while telephone visits were performed for those beyond 5 years of follow up. Six hundred and sixty-three patients wre enrolled into the study and followed for mean $( \pm$ SD) $5.3 \pm 2.0$ years. In the comparison of patent foramen ovale closure group versus antiplatelet only group, there was no stroke reported among the 238 patients in the patent foramen ovale closure group, whereas 14 of 235 patients in the platelet only group had stroke $(\mathrm{P}<0.001){ }^{16}$

The number needed to treat (NNT) to prevent one stroke is 17 at 5.3 years or 90 if considered annually. Among those who underwent patent foramen ovale closure, 14 patients $(5.9 \%)$ had procedural complication. Although, the rate of atrial fibrillation was higher in the patent foramen ovale closure group than in the antiplatelet-only group. Serious adverse events did not differ significantly between these 2 treatments group. In the analysis of antiplatelet group versus anticoagulant group, stroke occurred in 3 of 187 patients $(1.5 \%)$ with anticoagulant versus 7 of 174 patients $(3.8 \%)$ assigned to antiplatelet therapy alone $(\mathrm{P}=0.18)$. The bleeding complications and serious events did not differ significantly, probably as a result of the small number of participants in this comparison.

For young and middle aged persons with cryptogenic stroke associated with high risk patent foramen ovale there is now some randomized trial evidence to support 
patent foramen ovale closure namely Gore REDUCE trial. The investigator conducted a multinational randomized trial including patients with cryptogenic stroke and patent foramen ovale with right to left shunt. Approximately $81 \%$ had a moderate or large shunt. Patients were enrolled from 63 centers including 664 patients with cryptogenic stroke and patent foramen ovale. These patients were randomly assigned in a 2:1 ratio to undergo patent foramen ovale closure with antiplatelet therapy or antiplatelet therapy alone respectively. Of the 664 patients, 441 patients were randomized to patent foramen ovale closure group and 223 patients were assigned to the antiplatelet alone group. The primary outcomes were the percentage of patients with clinically evident stroke and combined incidence of clinical ischaemic stroke and silent brain infarction on imaging through at least 24 months follow up post-randomization. Median follow up was 3.2 years.

The investigators found that patients who underwent patent foramen ovale closure with antiplatelet therapy had a significantly lower incidence of clinical ischaemic stroke $(1.4 \%)$ as well as new brain infarction $(5.7 \%)$ as against antiplatelet therapy alone group who had $5.4 \%$ and $11.3 \%$ incidence of clinical ischaemic stroke and silent brain infarction respectively.

$6.6 \%$ of patients in the patent foramen ovale closure group had atrial fibrillation or flutter. Of which at least 415 persisted more than two weeks. The NNT to prevent recurrent clinical ischaemic stroke over 3.2 years was 25 . The NNT to prevent recurrent clinical ischaemic stroke per patient year based on this trial is 77 .

This randomized trial gives evidence regarding the efficacy of patent foramen ovale closure in reccurent stroke reduction in patients under 60 years of age with moderate to large interatrial shunts and cryptogenic stroke. ${ }^{16-17}$

Based on those trials, it needs more randomized trial data are required to consistently establish the efficacy and safety of patent foramen ovale closure in preventing recurrent stroke and to find high risk sub-group of patients who could benefit significantly from procedure.

\section{Conclusion}

Long term safety of patent foramen ovale closure procedure needs to be established given the fact there is increased incidence of atrial fibrillation or flutter in the patent foramen ovale closure group. Neurologist and stroke clinicians evaluating very high-risk patients for patent foramen ovale closure do now have some evidence that supports closure and refer the procedure to interventional cardiologist.

\section{References}

1. Saver Jl. Cryptogenic Stroke. N Engl J Med 2016;374:2065-2074.

2. Adam HP, Bendixen BH, Kappelle LJ,et al. Calssification of subtype of acute ischemic stroke. Definition for use in a multicenter clinical trial. TOAST.Trial of Org 10172 in Acute Stroke.Stroke 1993;24:35-41

3. Robert G, Hart MD, Catanase L, Perera KS, Ntaios G, Connoly SJ. Embolic stroke of Undetermined Source A Systemic Review and Clinical Update. Stroke 2017;48:867-872

4. OzdemirAO,TamayoA, MunozA, et.al.Cryptogenic stroke and patent foramen ovale:clinical clues to paradoxical embolism.J Neural Sci 2008;275:121-7

5. Almekhali MA, Wilton SB, Rabi DM, Ghali WA, Larenzati ti DL, Hill MD. Recurrent cerebral ischemi in medically treated patent foramen ovale. A meta-analysis Neurology 2009;73(2):89-97

6. Furlan Aj, Reisman M, Massaro J, Mauri L, Adams $\mathrm{H}$, Albers GW, Felberg R, Hermann H, Kar S, Landzberg M, Raizver A, Wechsler L. CLOSURE Investigator. Closure or medical therapy for cryptogenic stroke with patient with patent foramen ovale. N Engl J Med 2012:991-999

7. Saver Jl, Carrol JD, Thaler DE,et al. Long-term Outcome of Patent Foramen Closure or Medical Therapy aftre stroke. N Engl J Med 2017;377:10221032

8. Sandergraad L, Kasner SE, Rhodes JF. Paten Foramen Ovale or Antiplatelets Therapy for Cryptogenic Stroke. N Engl J med 2017;377:10331042 
9. Louis JM, Derumeaux G, Guillion B,et al. Patent Foraman Ovale Closure or Anticoagulant vs Antiplatelets after Stroke. N Engl J Med 2017;377:1011-1021

10. Tobe J, Bogitzi, Munoz C et all. Transcranial doppler is complimentary for detection and risk stratification of patent formane ovale. Can J Cardiol 2016;32:986.e9-986e16

11. Pristipino C, Sievert H, D'Ascenzo F, Louis Mas J, Meier B, Scacciatella P, et al. European position paper on the management of patients with patent foramen ovale. General approach and left circulation thromboembolism. Euro Heart J. 2019 ;40(38):3182-95.

12. Nakayama R, Takaya Y, Akagi T, Watanabe N, Ikeda M, Nakagawa K, et al. Identification of High-Risk patent foramen ovale Associated With Cryptogenic Stroke: Development of a Scoring System. Journal of the American Society of Echocardiography : official publication of the American Society of Echocardiography. 2019 ;32(7):811-6.

13. Glutzer TV, Zigler PD: Cryptogenic stroke: Is silent atrial fibrillation on the culprit ? Heart Rhythm 2015;12:234-241

14. Morais LA, de Sousa L, Fiarresga, et al. RoPE Score as a Predictor od Recurrent Ischmeic Events After Percutaneous patent foramen ovale Closure.Int Heart J 2018;59:1327-1332

15. Garg A, Thawabi M, Rout A,Sossou C, Cohen M, Kosits JB. Recuurent Stroke Redustion with patent foramen ovale Colure versus Medical Therapy Based on patent foramen ovale Characteristics: A Meta-Analysis of Randomized Controlled Trials. Cardiology 2019:144(1-2):40-49.

16. Vaduganathan M, Qamar rman, Gupta,et.al. Paten Foramen Ovale Closure for Secondary Prevention of Cryptogenic Stroke: Updated Metaanalysis of Randomized Clinical Trials. Am J Med 2018:131(5):575-577.

17. Giblett JP, Abdul-Samad O, Shapiro LM, Rana BS, Calvert PA. patent foramen ovale Closure in 2019. Interventional cardiology. 2019 Feb;14(1):34-41 